\title{
A Comprehensive Review of Alternative Therapies for the Management of Chronic Pain Patients: Acupuncture, Tai Chi, Osteopathic Manipulative Medicine, and Chiropractic Care
}

\author{
Ivan Urits · Ruben H. Schwartz • Vwaire Orhurhu • Nishita V. Maganty • \\ Brian T. Reilly · Parth M. Patel · Christopher Wie · Alan D. Kaye • \\ Ken F. Mancuso · Aaron J. Kaye · Omar Viswanath
}

Received: September 8, 2020 / Accepted: October 28, 2020 / Published online: November 12, 2020

(C) The Author(s) 2020

\section{ABSTRACT}

Introduction: Non-pharmacologic alternative therapies for pain have been around for a long time, some for hundreds of years. They have been used throughout history to treat many issues.

Recent Findings: Currently, alternative medicine is most frequently used to treat musculoskeletal pain, and between 59 and $90 \%$ of

I. Urits $(\bowtie) \cdot$ V. Orhurhu

Department of Anesthesiology, Critical Care and

Pain Medicine, Beth Israel Deaconess Medical

Center, Harvard Medical School, Boston, MA, USA

e-mail: ivanurits@gmail.com

R. H. Schwartz

Department of Anesthesiology, Mount Sinai

Medical Center, Miami Beach, FL, USA

N. V. Maganty · P. M. Patel

University of Arizona College of Medicine-Phoenix, Phoenix, AZ, USA

B. T. Reilly

Creighton University School of Medicine, Phoenix

Regional Campus, Phoenix, AZ, USA

C. Wie

Department of Anesthesiology, Mayo Clinic

Arizona, Phoenix, AZ, USA

A. D. Kaye · K. F. Mancuso

Department of Anesthesiology, Louisiana State

University Health Sciences Center, New Orleans, LA, USA patients utilizing alternative therapies for chronic pain claimed they were helpful and can serve as an effective adjunctive for the treatment of chronic pain. Some examples of alternative therapies that will be discussed in this review include acupuncture, tai chi, osteopathic manipulation, and chiropractic care. Acupuncture, traditionally a Chinese practice, is becoming more popular across the world to attempt to relieve pain. It involves the placement of thin needles at various points in the
A. J. Kaye
Department of Anesthesiology, Medical University
of South Carolina, Charleston, SC, USA
O. Viswanath
Department of Anesthesiology, University of Arizona College of Medicine-Phoenix, Phoenix, AZ, USA
O. Viswanath
Department of Anesthesiology, Creighton University
School of Medicine, Omaha, NE, USA
O. Viswanath
Valley Anesthesiology and Pain Consultants,
Phoenix, AZ, USA 
body. The efficacy of acupuncture for pain is heavily debated. More research and discussion are necessary to determine the exact role it plays in the treatment of chronic pain. Tai chi is also a traditional Chinese practice that is often used as a form of meditation and for potential health benefits. Tai chi involves a series of complex movements such as squatting combined with deep breathing to achieve relaxation and pain reduction. Osteopathic manipulative treatment (OMT) is a technique used by both osteopathic physicians (DO) as well as other health professionals to manage a wide range of conditions in any given patient. The technique involves utilization and manipulation of the musculoskeletal system to achieve potential health benefits. OMT has been used as therapy for many issues but is commonly used for pain conditions.

Summary: Alternative therapies may serve as an effective adjunctive treatment modality for the management of chronic pain conditions. There has been a tremendous amount of research dictating the effectiveness of alternative therapies for chronic pain management. The purpose of this review is to provide a comprehensive evidence-based update of alternative therapy used for the management of chronic pain conditions.

Keywords: Acupuncture; Chiropractor; Osteopathic manipulative treatment; Pain; Tai chi

\section{Key Summary Points}

Alternative therapies provide evidencebased options for patients dealing with chronic pain to avoid unwanted opioid use.

These techniques have been utilized for centuries with proven results.

Tai chi, acupuncture, and osteopathic manipulation therapy are all viable options for improving mobility and reducing pain symptomatology.

\section{DIGITAL FEATURES}

This article is published with digital features, including a summary slide, to facilitate understanding of the article. To view digital features for this article go to https://doi.org/10.6084/ m9.figshare.13142822.

\section{INTRODUCTION}

Pain occurs in $30.7 \%$ of adults [1]. Between 9 and 12 million people in the US suffer from chronic pain [2]. Chronic pain is incredibly debilitating and comes in many different forms. Some different types of chronic pain include neuropathic, nociceptive, and nociplastic pain. It is associated with decreased function both physically and emotionally as well as a decrease in quality of life [3]. Additionally, the total cost burden due to chronic pain in the US is estimated to be between $\$ 560$ and $\$ 635$ billion dollars annually [3]. There are many ways to manage pain. Pharmaceutical therapy is usually the first choice with opioid therapy often being considered for severe chronic pain. Patients that require opioids for pain relief usually have exhausted more conservative analgesics such as non-steroidal anti-inflammatories and acetaminophen. Opioids, despite being highly addictive, are still one of the most prescribed pharmaceuticals for this particular issue. There are five opioid medications in the top 200 most prescribed drugs in the US and one within the top 10 [4]. The increase in opioid use in the US is multifactorial. However, many attribute the increase over the years to both the introduction of pain as the fifth vital sign in the 1990s and the aggressive marketing of OxyContin by Purdue Pharma [5, 6]. A 2019 literature review noted that around $6 \%$ of the US abused opioids in some form [2]. Prescription opioids are often described as a "gateway into the drug culture" [7]. The rise in prescription opioid abuse has been linked to increases in heroin and synthetic opioid (fentanyl, etc.) related deaths with a $20 \%$ and $72 \%$ bump in overdose deaths as a result of these substances, respectively [5]. Deaths from opioid overdose have risen $156 \%$ from 2010 to 
2015 [8]. There are alternative medications available to treat daily pain. NSAIDs are one of the most common classes of non-opioid pain medication. These drugs may not adequately control pain and are associated with increased risk of gastrointestinal ulcers and bleeding $[9,10]$. Several other classes of drugs such as antidepressants and muscle relaxants are often prescribed for pain, but have weak evidence for efficacy against chronic pain [10]. Non-pharmacologic alternative therapies for pain have been around for a long time, some for hundreds of years. They have been used throughout history to treat many issues. Currently, alternative medicine is most frequently used to treat musculoskeletal pain and between 59 and $90 \%$ of patients utilizing alternative therapies for chronic pain claimed them to be "helpful" [11]. Based on these findings, it appears that alternative therapies can serve as an effective adjunctive for the treatment of chronic pain.

Some examples of alternative therapies that will be discussed in this review include acupuncture, tai chi, osteopathic manipulation, and chiropractic care. Acupuncture, traditionally a Chinese practice, is becoming more popular across the world to attempt to relieve pain. It involves the placement of thin needles at various points in the body. A 2012 meta-analysis of nearly 18,000 patients found acupuncture to be better than sham and no acupuncture therapy for chronic pain control [12]. However, the efficacy of acupuncture for pain is heavily debated. More research and discussion are necessary to determine the exact role it plays in the treatment of pain [13]. Tai chi is also a traditional Chinese practice that is often used as a form of meditation and for potential health benefits. Tai chi involves a series of complex movements such as squatting combined with deep breathing to achieve relaxation and pain reduction [14]. An overview of current Cochrane reviews regarding physical activities and chronic pain suggests that interventions such as tai chi are potentially beneficial in reducing chronic pain and increasing quality of life; however, additional research is needed before specific recommendations can be made [15]. Osteopathic manipulative treatment (OMT) is a technique used by both osteopathic physicians (DO) as well as other health professionals to manage a wide range of conditions in any given patient. The technique involves utilization and manipulation of the musculoskeletal system to achieve potential health benefits. OMT has been used as therapy for many issues but is commonly used for pain conditions. A systematic review of OMT for low back and pelvic pain after surgery showed evidence suggesting moderate reduction in pain for these conditions following OMT [16]. Chiropractic care is a practice founded in the 1890s that is a well-established form of alternative medicine in the western world. Like OMT, it focuses on manual manipulation of the body to treat issues with the musculoskeletal system. Chiropractic care is very controversial, especially in the discussion of chronic pain. There is a clear need to step away from opioids in the management of chronic pain given the current epidemic. Alternative therapies are needed to aid in management of chronic pain so there is a reduced focus on opioid pain management. Chronic pain, unlike acute pain, often does not have a discernable physiologic cause or pathology [17]. Therefore, alternative therapies such as those discussed above may serve to be a reasonable alternative to opioids for management of chronic pain conditions. We focused our review on the most prevalent form of pain in the patient population, low back pain. In this review, we will be discussing the benefit of utilizing alternative medicine approaches to the patient with chronic pain. This article is based on previously conducted studies and does not contain any studies with human participants or animals performed by any of the authors.

\section{ACUPUNCTURE}

\section{Low Back Pain}

Acupuncture, a form of alternative medicine that originated in China over 3000 years ago, has been increasingly used for pain management. There are a number of forms of acupuncture including needling, moxibustion, and cupping [18]. Needling involves inserting needles, typically 32-36 gauge, into specific 
acupuncture points on the body. Needles can be made of ceramic, bronze, iron, gold, silver, or stainless steel. In electroacupuncture, a current is applied through the needle. Laser acupuncture uses non-thermal laser irradiation on standard acupuncture points. Moxibustion utilizes burning moxa above the skin near pressure points. Finally, cupping is a technique in which bamboo, glass, or ceramic cups are used to create a vacuum over the acupuncture points [19]. Nearly 400 acupoints have been describes worldwide, located on nearly 20 meridians, or channels of energy. The goal of acupuncture is to promote flow of Qi, the essence of life, through the meridians [20].

Although acupuncture has been used in a variety of clinical disorders, it is most frequently used for chronic pain management. Acupuncture is beneficial for low back pain up to 2 years after treatment. In a randomized controlled trial (RCT) of 241 patients aged 18-64, acupuncture was found to have a significantly favorable effect on the SF-36. Patients who had received ten acupuncture sessions over 3 months had a 5.6-point increase on the SF-36 at 12 months and an 8.0-point increase at 24 months compared to those receiving standard treatment. Furthermore, the patients that received acupuncture reported lower scores on the Oswestry Disability Index (ODI) and McGill pain measure, although this was not statistically significant. There were no severe adverse effects of acupuncture reported in this study [21]. In another RCT, 130 adults with low back pain were randomized to receive acupuncture or sham acupuncture twice weekly for 6 weeks. Patients who received true acupuncture reported lower scores for pain compared to those that received sham acupuncture. Sham acupuncture in this study was defined by using blunt needles without penetration on acupuncture points. This demonstrates that acupuncture has more than a placebo psychologic effect and there is a physiologic mechanism underlying its success [22]. In addition, standard acupuncture therapy is shown to be equally effective to individualized therapy for patients [23]. Electroacupuncture has been shown to be effective in the treatment of chronic sciatica. In a study of 100 patients randomized to receive electroacupuncture versus medium frequency electrotherapy, there was a significant improvement in pain based on ODI scores in the electroacupuncture group [24]. In addition, results of a randomized double-blind trial illustrated that laser acupuncture using cups with a frequency of $200 \mathrm{~Hz}$ and output power of $50 \mathrm{~mW}$ resulted in similar pain reduction and quality of life scores as a sham laser cupping control. This demonstrates that cupping itself may provide a therapeutic effect for low back pain. One limitation of the study was that laser therapy was only studied for three treatments [25].

\section{Pregnancy}

Acupuncture may also be a useful treatment in pregnancy-related low back pain. The prevalence of low back pain during pregnancy is estimated to be between 30 and 70\% [26]. Back pain in pregnancy is due to a combination of hormonal, mechanical, and motor changes. An enlarging uterus to carry a growing fetus causes increased strain on the lumbar spine, which results in low back pain. The enlarging uterus causes abdominal muscles to stretch significantly resulting in a decrease in strength and coordination of the lumbopelvic muscles, particularly the gluteus medius. Finally, a hormone released by the placenta called relaxin is thought to weaken joint strength, resulting in low back pain [27]. Acupuncture is an emerging treatment for pregnancy-related low back pain. In the EASE back pilot trial, 125 pregnant patients were randomized to receive standard care for back pain, standard care plus sham acupuncture, or standard care plus true acupuncture. After six treatments over 8 weeks, patients receiving sham acupuncture and true acupuncture endorsed improvement in pain and physical health. Patients reported no difference in quality of life but stated that they had fewer nighttime awakenings and sleep disturbances due to back pain. Earlier studies suggested that acupuncture may lead to pre-term labor in pregnant patients; however, recent literature, including the EASE back trial, reported no serious adverse events associated with 
acupuncture in pregnancy. The most common side effects include lightheadedness and pain at the acupuncture site [26]. A Cochrane review published in 2015 reported that there was no significant difference in sham acupuncture with prenatal care and true acupuncture with prenatal care in pregnant patients with pelvic pain. However, compared to prenatal care alone, acupuncture results in moderate improvement in pelvic pain [28]. Acupuncture may also have utility in improving labor pain. A Cochrane review published in 2011 stated acupuncture used at variety of acupoints resulted in a reduction of labor pain. Patients were also more satisfied with acupuncture as a pain treatment compared to standard treatment [29]. Two studies published in 2011 demonstrated that patients who received electroacupuncture during labor had a significant reduction in pain [30].

\section{Migraines}

Acupuncture is a useful treatment in the management of migraines. A 2016 Cochrane review evaluated 22 RCTs with over 4000 participants and found that acupuncture resulted in a $50 \%$ reduction of migraines in $41 \%$ of the patients. Acupuncture was superior to sham acupuncture in reduction of migraines, and a small benefit was found even 12 months after treatment [31]. In a study of 20 women suffering from chronic migraines, ear acupuncture resulted in a reduction of migraine attacks per month in $37.5 \%$ of women [32]. When combined with tuina therapy, a form of therapeutic massage, acupuncture may be more effective in treating migraines. In an RCT of 45 patients, those receiving tuina therapy combined with acupuncture compared with those receiving acupuncture alone or control therapy had a significant reduction in attack frequency, severity of pain, and duration of migraine. The combination of tuina with acupuncture resulted in less analgesic use by patients in the trial [33]. Although acupuncture appears to be effective in relieving migraines, its mechanism of action is not well understood. In an animal study, acupuncture resulted in a decrease in the expression of 5-HT7R, a serotonin pathway, and subsequent alleviation of migraine symptoms [34]. Magnetic resonance spectroscopy pre- and post-acupuncture found a significant increase in $N$-acetylaspartate and creatine in the thalamus [35]. Future studies are necessary to better understand the mechanism behind acupuncture and duration of symptom relief.

\section{Complex Regional Pain Syndrome (CRPS)}

Complex regional pain syndrome (CRPS) is a chronic neuropathic pain syndrome believed to be caused by inflammation or damage to the nervous system after an injury. CRPS is commonly managed with NSAIDs, steroids, antidepressants, and regional pain blocks. It can often be resistant to these forms of standard pain treatment. Acupuncture may be an important treatment option for CRPS. In a case report of a 29-year-old man with CRPS after polydactyly resection, bee venom acupuncture resulted in significant reduction in pain. After 14 over 3 months, the patient reported complete resolution of pain symptoms and no longer needed analgesic medications [36]. In another case report, ultrasound-guided dry needling resulted in almost complete improvement of pain and motor dysfunction within 2 months of treatment. In animal studies, electroacupuncture is thought to work by inhibiting mechanical hyperalgesia and edema in chronic post-ischemia pain mouse models. This effect is enhanced when electroacupucture is used in conjunction with endothelin receptor type B (ETB) antagonists [37].

The efficacy of acupuncture for chronic pain conditions is summarized in Table 1.

\section{TAI CHI}

\section{Low Back Pain}

Tai chi is a Chinese mind-body exercise that has been used for health promotion and disease prevention in Asia for hundreds of years. In America, the prevalence of tai chi practice has grown steadily since 2002, with a $14.5 \%$ 
Table 1 Efficacy of acupuncture in the treatment of chronic pain conditions

Low back pain
$\begin{aligned} & \text { Acupuncture is superior to traditional pain management in patients with low back pain. } \\ & \text { The number of sessions required for therapeutic effect is varied }\end{aligned}$
$\begin{aligned} & \text { Acupuncture and sham acupuncture are important treatment options in pregnancy-related } \\ & \text { back pain and labor }\end{aligned}$
$\begin{aligned} & \text { Acupuncture may result in a reduction of the frequency and intensity of migraine } \\ & \text { headaches. Larger cohort studies are needed to analyze this further }\end{aligned}$
$\begin{aligned} & \text { Complex regional pain } \\ & \text { syndrome (CRPS) }\end{aligned}$

prevalence in 2017. Tai chi practice has increased in each demographic across the US. Most people report using tai chi for overall wellness; however, approximately $15 \%$ of people use tai chi to help with specific medical conditions. Tai chi practice is usually used for pain, arthritis, and mental health [38]. In a randomized controlled trial of 320 retired athletes with chronic lower back pain, tai chi practice resulted in reduction of pain compared to no exercise and jogging, but had no significant difference compared with swimming [39]. In another randomized controlled trial of 160 people, tai chi was performed 18 times over 10 weeks and led to a reduction of pain and bothersome back pain and improved self-reported disability based on the Roland-Morris Disability Questionnaire scale. This study focused on a 10-week trial of tai chi; however, future studies are needed to understand the long-term impact of tai chi on low back pain [40]. In addition, the broader implications of tai chi practice such as the effect on daily physical activity remain to be studied. A 12-week tai chi intervention for low back pain resulted in increased knee extension and right plantar flexion compared to core stability training. This demonstrates that tai chi may have beneficial neuromuscular effects in addition to pain reduction [41].

\section{Elderly Patients}

Older individuals may also benefit from tai chi practice. In a randomized trial of 43 adults over age 50 with chronic lower back pain, Chen-style tai chi led to a reduction in pain on the visual analog scale compared to core stabilization training. There was no difference in lower limb proprioception in tai chi versus core stabilization training. Chen-style tai chi places greater emphasis on alternating between fast and slow movements, which is thought to increase lumbar spine stimulation and improve lower back strength. Core stabilization training utilizes a Swiss ball to perform specific core-strengthening exercises [42]. In older individuals with multiple comorbid conditions including HIV and substance abuse, tai chi may improve physical function and reduce substance use. In one study, 55 adults with a mean age of 55 who had HIV and substance abuse were randomized to receive cognitive behavioral therapy (CBT) with tai chi, support group therapy, or control. Individuals in the CBT followed by tai chi group reported a reduction in pain intensity, decrease in substance use, and improvement in physical performance outcomes [43]. In the patients that were required to attend CBT and tai chi, attendance was less than compared to support group therapy alone, which suggests that individuals may need to be motivated to attend both.

\section{Multiple Sclerosis}

Multiple sclerosis (MS) is an autoimmune neurodegenerative disease that affects the central nervous system. Patients with MS can have a variety of neurologic symptoms including ataxia, impaired balance, and tremors. In 
addition, they also have higher reported rates of pain, depression, and fatigue. Given that tai chi is a mind-body exercise it can have valuable effects on both physical and psychologic conditions. In a study of 32 MS patients, tai chi practiced twice weekly for 6 months resulted in significant improvement in balance and coordination. To assess balance and coordination, two well-established tests consisting of 14 tasks and 10 tasks, respectively, were utilized. In addition to the physical improvements, patients who were in the tai chi group had lower selfreported scores for depression and fatigue. This study shows that tai chi may be a promising intervention for MS patients improving both physical and psychologic parameters [44]. However, this study had a small sample size and relatively long intervention period; therefore, repeating the study in a larger cohort with a shorter duration may be helpful. Tai chi has also demonstrated significant benefits for depression, quality of life, and motor control [45]. In an 8-week, prospective, nonrandomized controlled trial, 40 patients with MS or peripheral neuropathy participated in either a mediation group or standard care. Patients who were in the mediation group, which consisted of tai chi, had significant improvement in pain following weekly 30-min meditation classes [46]. This study was limited by the small sample size and lack of randomization.

\section{Post-Traumatic Stress Disorder (PTSD)}

Post-traumatic stress disorder (PTSD) is a psychiatric condition that occurs in individuals who have experienced or witnessed trauma. It is characterized back recurrent flashbacks, nightmares, and hypervigilance. Often patients with PTSD also suffer from chronic musculoskeletal pain generally in the back and neck. PTSD is traditionally treated with CBT; however, this may not address the chronic pain that these patients suffer. Tai chi may be a useful tool in improving chronic pain in patients with PTSD. In a study of 11 patients with PTSD, $90.0 \%$ of individuals reported their health would improve with tai chi. After completing 36 tai chi sessions over 3 months, participants reported a reduction in current pain based on the PROMIS pain intensity score. There was a decrease in use of pain medications before and after the intervention although this was not statistically significant. Some participants noted that tai chi made them feel pain or discomfort while practicing; however, no other adverse events were reported [47]. In a case report of 44-year-old Lebanese man with PTSD, tai chi resulted in marked improvement in back and neck pain. In the future, larger studies are needed to evaluate the effect of tai chi in PTSD patients with chronic pain [48].

The efficacy of tai chi for the treatment of chronic pain conditions is summarized in Table 2 .

\section{OSTEOPATHIC MANIPULATIVE MEDICINE}

Osteopathic manipulative medicine (OMT) is the usage of physical techniques by trained physicians that are intended to help facilitate

Table 2 Efficacy of tai chi in the treatment of chronic pain conditions

Low back pain

Older patients

Multiple sclerosis

Post-traumatic stress disorder (PTSD)
Short-term, tai chi reduces pain in chronic lower back pain

Tai chi improves back pain in patients $>$ age 50 and those with comorbid conditions such as HIV and substance abuse

There is minimal evidence that tai chi improves pain in MS patients. Several studies demonstrate improvement in balance, coordination, depression, and fatigue

Tai chi may be beneficial in patients with PTSD and musculoskeletal pain 
the healing of patients. The Journal of Family Practice defines OMT as "the process by which DOs use their hands to diagnose illness and injury and then mobilize a patient's joints and soft tissues using techniques that include muscle activation, stretching, joint articulation, and gentle pressure to encourage the body's natural tendency to heal itself" [49]. However, it is important to note that OMT is not restricted to osteopathic physicians, as allopathic physicians can undergo additional training if they wish to use it in their own practice. The American Osteopathic Association (AOA) encourages the use of OMT by trained physicians [50]. With the growing number of osteopathic medical schools in the United States, the number of physicians with the training to offer OMT is rising. Currently, over $20 \%$ of medical students attend an osteopathic medical school [49].

Although the AOA's recommendation is based on evidence-based published literature, it is not without criticism. Critics of OMT point to the subjectivity of the quality of treatment offered by different physicians, as it can vary greatly depending on the physician's training, experience, and frequency with which the perform OMT. Additionally, people are critical of the lack of universality of the recommended techniques. Many osteopathic physicians have become dissuaded from using OMT because of the poor financial reimbursement, its time-intensive nature, and/or its lack of applicability in different fields of practice, such as radiology and pathology [50]. This section aims to examine the efficacy of OMT in the treatment of lower back pain and migraines.

\section{Low Back Pain}

Given that low back pain is the most common cause of disability worldwide, the effectiveness of OMT in the treatment of LBP warrants investigation. In 2016, Licciadarone, Gatchel, and Aryal published the results of a randomized controlled trial on the effectiveness of OMT in the treatment of LBP. The study was designed to resemble a 2011 publication titled "Osteopathic Health Outcomes in Chronic Low Back Pain (OSTEOPATHIC) Trial." The 2011 publication was also a RCT, but contained only 145 patients. The 2016 study contained 345 patients, thus increasing the power of the study.

In the 2016 study, patients with LBP were randomly assigned using a computer-generated algorithm to receive either OMT or sham OMT. They then received treatment at weeks $0,1,2,4$, 6 , and 8 followed by an outcome assessment at week 12 . Outcomes were defined by a $100-\mathrm{mm}$ visual analog scale (VAS) and the Roland-Morris Disability Questionnaire (RMDQ). In total, 23 patients enrolled in the study experienced adverse events, but none were determined to have been caused by study interventions. The results of the study showed a statistically significant difference between the two groups. The number needed to treat (NNT) for patients who underwent OMT was 9.9 (95\% CI 5.8-36.2.) However, this study was limited by its inclusion criteria, which required patients to meet minimum thresholds of pain using the VAS or RMDQ. As such, the usage of OMT for the treatment of patients with minor LBP remains unclear [51]. Additionally, the study is limited by its limited follow-up of participants. While the study demonstrates a statistically significant difference at 12 weeks, it fails to examine the long-term outcomes of the patients. Given the time-consuming nature of OMT, it may be difficult for patients to continue to receive treatment on a weekly or biweekly basis. Thus, the long-term efficacy of OMT in the treatment of LBP remains unclear and warrants further research.

\section{Migraine}

While OMT continues to be offered by osteopathic physicians for the treatment of migraine headaches, its efficacy is more controversial than in the treatment of LBP. A 2015 randomized controlled trial that included 105 patients revealed a statistically significant improvement in functional improvement of patients who underwent OMT for the treatment of migraine headaches. Patients who underwent OMT experienced a reduction in HIT-6 score, drug consumption, number of days with migraine(s), pain intensity, and functional disability [52]. 
Table 3 Efficacy of osteopathic manipulation in the treatment of chronic pain conditions

\begin{tabular}{c}
$\begin{array}{c}\text { Low back } \\
\text { pain } \\
\text { although data regarding its long-term } \\
\text { efficacy are yet to be published }\end{array}$ \\
Migraines $\quad$ Low level of evidence to support its efficacy \\
\hline
\end{tabular}

With a patient sample size of just 105 patients, further studies are required to increase the power of this evidence.

In 2017, Cerritelli et al. performed a thorough systematic review that aimed to further assess the effectiveness of OMT in the treatment of patients with migraine headaches. Using five of the six studies available at that time, the authors concluded that there is a "low level of evidence that OMT is effective in the management of headache." However, they acknowledged that more thorough studies are required to be able to come to a more agreed-upon conclusion [52].

The efficacy of osteopathic manipulation for the treatment of chronic pain conditions is summarized in Table 3.

\section{CHIROPRACTIC}

\section{Low Back Pain}

In 2017, Goertz et al. published the results of a randomized-controlled trial studying the efficacy of chiropractic manipulation in the treatment of LBP. In this study, patients were assigned to one of three cohorts: medical care only, medical and chiropractic care, or medical and chiropractic care with additional interprofessional collaboration. Potentially eligible patients were contacted electronically or via mail. Patients were required to have experienced at least 1 month of LBP and needed to rate the pain as at least a 4 on an 11-point scale. One hundred thirty-one subjects participated in the trial. The results of this study showed improvements in pain and back-related disability in each of the three groups. None of the groups appeared to be statistically significant from the others in these measurements.
However, the two groups receiving chiropractic manipulation experienced superior improvements in perceived pain and were more satisfied with their treatment compared to the group that received medical management alone [53]. This study is limited by its small sample size as well as selection bias. With just 153 patients involved, it is difficult to come to a consensus on the effectiveness of chiropractic manipulation in the treatment of LBP. Additionally, because patients were recruited using mail and media, a selection bias was inherent in the study. To determine the applicability of these results to the general population, additional studies will need to be performed.

\section{Migraine}

Despite a lack of clinical evidence supporting its use, chiropractic manipulation continues to be commonly used in the treatment of migraine headaches. A 2017 study by Moore et al. showed that $53.0 \%$ of chiropractors use manipulation to treat patients "often," $40.9 \%$ use it "sometimes," $5.7 \%$ use it "rarely," and only $0.4 \%$ use it "never." Given the lack of evidence to support its applicability in this population of patients, these statistics are surprising. However, given that migraine headaches are one of the leading causes of disability worldwide, there is no shortage of patients to be seen with this condition. Many patients prefer a non-medicinal approach to treatment and thus view chiropractic manipulation as a viable approach. Given the lack of evidence to support its use in this patient population, alternative approaches should be considered.

According to Moore et al., "The high use of chiropractors by those with migraine would suggest these providers are likely to be addressing some of the healthcare needs of this population" [54]. However, what this statement fails to acknowledge is that the demand for a specific therapy does not directly correlate to its efficacy. The placebo effect is a well-understood concept, and its existence is highly accepted by the medical community. As a result, one cannot deduce that simply because patients are using chiropractors to treat their migraines their 
treatment must be effective. To address this issue, a randomized placebo-controlled trial must be performed.

In 2016, Chaibi et al. showed that such a study can be performed. Although their patient sample size was just 104, the results of their study showed no significant difference between patients who had received chiropractic manipulation or sham therapy [55]. Without a randomized controlled trial that contraindicates these data, the use of chiropractic manipulation in the treatment of patients with migraine headaches is a poor use of resources. Additionally, with the principle of beneficence in mind, providers must question whether providing services to patients that have been shown to be effective solely because of the placebo effect is ethical.

\section{Neck Pain}

The use of chiropractic practices in the treatment of patients with acute neck pain remains poorly studied. As a result, the recommendations remain unclear. In 2016, Bussieres et al. published guidelines based upon six previously published systematic reviews on the topic. Regarding patients with recent-onset (0-3 months) neck trauma, they supported the usage of manipulation if the patient desired such treatment. However, they acknowledged that this recommendation was based upon lowquality evidence. This was largely due to the short follow-up time of the patients involved in the main study they used to make this recommendation. Patients in this study were treated four times over the course of 2 weeks and monitored using the Kaplan-Meier recovery curve. Patients were randomly assigned to receive their treatment from physiotherapists, chiropractors, or osteopaths and were required to have grade I or II neck pain. Although they found that there was no statistically significant difference between the different groups, one cannot deduce from this that chiropractic manipulation is an effective treatment for neck pain. Doing such assumes that manipulation in general aids in the recovery of neck pain, which is not necessarily true.
Table 4 Efficacy of chiropractic manipulation in the treatment of chronic pain conditions

\begin{tabular}{|c|c|}
\hline $\begin{array}{l}\text { Low back } \\
\text { pain }\end{array}$ & Low level of evidence to support its efficacy \\
\hline Migraines & $\begin{array}{l}\text { Highly popular, although no evidence to } \\
\text { support its efficacy }\end{array}$ \\
\hline Neck pain & $\begin{array}{l}\text { Efficacy remains unclear for acute and } \\
\text { chronic neck pain }\end{array}$ \\
\hline
\end{tabular}

Regarding patients with chronic neck pain (> 3 months), Bussieres et al. reached a similar conclusion. This conclusion was the result of two previous publications comparing patients with grades I and II back pain who received one manipulation session per week for 20 weeks and those who did not. One of the two studies showed a decrease in pain level at the 12-week mark. However, neither study showed a statistically significant difference between the two groups at 52 weeks. From these studies, Bussieres et al. recommended the use of manipulation in the treatment of persistent neck pain. Once again, they acknowledged that this recommendation is weak and supported by lowquality evidence. Additionally, they acknowledged that the potential benefits and consequences of manipulative therapy were roughly equal. With this in mind, manipulative therapy in the treatment of persistent neck pain appears to be a poor use of resources and other forms of therapy should be considered (56).

The efficacy of chiropractic therapy for the treatment of chronic pain conditions is summarized in Table 4.

\section{CONCLUSION}

Chronic pain is an issue that affects millions of patients daily. Current treatments usually include pharmacologic therapies, most notably opioid therapy. However, chronic opioid use carries immense risk given the potential for overdose and addiction with this class of medications. With the current crisis surrounding opioid use and increases in opioid-related deaths in the US, there is a clear need for 
alternative treatment of chronic pain. Many of the pharmacologic alternatives to opioids either are not as effective for chronic pain or carry their own risks and adverse effects such as in increased risk of gastrointestinal bleeding. Other modalities for treating chronic pain include therapies such as acupuncture, tai chi, OMT, and chiropractic care, which were all evaluated in this review as alternative and holistic approaches to managing chronic pain. Acupuncture was shown to be effective in reducing chronic low back pain and pregnancyrelated pain. However, they were ultimately inconclusive in their use for migraines and CRPS. Tai chi, similarly, was shown to be useful in chronic low back pain, specifically in the older population. However, it was ineffective for MS-related pain and PTSD. OMT was shown to be useful in management of low back pain but not for migraine. Chiropractic manipulation was not shown to be effective in management of low back pain, migraine, or neck pain. Overall, the evidence for these alternative therapies in the management of chronic pain is mixed. While there is some evidence to claim that specific therapies can be effective for specific types of pain (for example, acupuncture for low back pain), there was also evidence available to refute that claim. At this point in time we cannot conclude that alternative therapies are a replacement for pharmaceutical management of chronic pain. However, they may play a role as adjuvant therapies that can potentially reduce a patient's overall opioid need. Additional longitudinal studies are needed to evaluate the role of alternative therapies in the context of chronic pain management.

\section{ACKNOWLEDGEMENTS}

Funding. No funding or sponsorship was received for this study or publication of this article.

Authorship. All named authors meet the International Committee of Medical Journal Editors (ICMJE) criteria for authorship for this article, take responsibility for the integrity of the work as a whole, and have given their approval for this version to be published.

Disclosures. Ivan Urits is a member of the journal's Editorial Board. Ruben H. Schwartz, Vwaire Orhurhu, Nishita V Maganty, Brian T. Reilly, Parth M. Patel, Christopher Wie, Alan D. Kaye, Ken F. Mancuso, Aaron J. Kaye, and Omar Viswanath have nothing to disclose.

Compliance with Ethics Guidelines. This article is based on previously conducted studies and does not contain any studies with human participants or animals performed by any of the authors.

Open Access. This article is licensed under a Creative Commons Attribution-NonCommercial 4.0 International License, which permits any non-commercial use, sharing, adaptation, distribution and reproduction in any medium or format, as long as you give appropriate credit to the original author(s) and the source, provide a link to the Creative Commons licence, and indicate if changes were made. The images or other third party material in this article are included in the article's Creative Commons licence, unless indicated otherwise in a credit line to the material. If material is not included in the article's Creative Commons licence and your intended use is not permitted by statutory regulation or exceeds the permitted use, you will need to obtain permission directly from the copyright holder. To view a copy of this licence, visit http://creativecommons.org/licenses/by$\mathrm{nc} / 4.0 /$.

\section{REFERENCES}

1. Bauer BA, Tilburt JC, Sood A, Li G, Wang S. Complementary and alternative medicine therapies for chronic pain. Chin J Integr Med. 2016;22(6): 403-11.

2. Stoicea N, Costa A, Periel L, Uribe A, Weaver T, Bergese SD. Current perspectives on the opioid crisis in the US healthcare system. Medicine. 2019;98(20):e15425. 
3. Stanos S, Brodsky M, Argoff C, Clauw DJ, D'Arcy Y, Donevan S, Gebke KB, Jensen MP, Lewis Clark E, McCarberg B, Park PW, Turk DC, Watt S. Rethinking chronic pain in a primary care setting. Postgrad Med. 2016;128(5):502-15.

4. Fuentes AV, Pineda MD, Venkata KCN. Comprehension of top 200 prescribed drugs in the US as a resource for pharmacy teaching, training and practice. Pharmacy (Basel). 2018;6(2):43.

5. Skolnick P. The opioid epidemic: crisis and solutions. Annu Rev Pharmacol Toxicol. 2018;58: 143-59.

6. Alam A, Juurlink DN. The prescription opioid epidemic: an overview for anesthesiologists. Can J Anesth. 2016;63(1):61-8.

7. Brown RE, Sloan PA. The opioid crisis in the United States. Anesth Analg. 2017;125(5):1432-4.

8. Gostin LO, Hodge JG, Noe SA. Reframing the opioid epidemic as a national emergency. JAMA. 2017;318(16):1539.

9. Labianca R, Sarzi-Puttini P, Zuccaro SM, Cherubino P, Vellucci R, Fornasari D. Adverse effects associated with non-opioid and opioid treatment in patients with chronic pain. Clin Drug Investig. 2012;32(Suppl 1):53-63.

10. Kroenke K, Cheville A. Management of chronic pain in the aftermath of the opioid backlash. JAMA. 2017;317(23):2365.

11. Simpson CA. Complementary medicine in chronic pain treatment. Phys Med Rehabil Clin N Am. 2015;26(2):321-47. https://doi.org/10.1016/j.pmr. 2014.12.005.

12. Vickers AJ, Cronin AM, Maschino AC, Lewith G, Macpherson H, Victor N, Foster NE, Sherman KJ, Witt CM. Acupuncture for chronic pain: individual patient data meta-analysis. Arch Intern Med. 2012;172(19):1444-53. https://doi.org/10.1001/ archinternmed.2012.3654.

13. Patil S, Sen S, Bral M, Reddy S, Bradley KK, Cornett EM, Fox CJ, Kaye AD. The role of acupuncture in pain management. Curr Pain Headache Rep. 2016;20(4):22.

14. Hall A, Copsey B, Richmond H, Thompson J, Ferreira M, Latimer J, Maher CG. Effectiveness of tai chi for chronic musculoskeletal pain conditions: updated systematic review and meta-analysis. Phys Ther. 2017;97(2):227-38.

15. Lj G. Cochrane database of systematic reviews physical activity and exercise for chronic pain in adults: an overview of cochrane reviews (review) physical activity and exercise for chronic pain in adults: an overview of cochrane reviews (review). Cochrane Database Syst Rev. 2017;1(1):CD0 11279. https://doi.org/10.1002/14651858.CD011279. pub2.

16. Franke H, Franke J-D, Belz S, Fryer G. Osteopathic manipulative treatment for low back and pelvic girdle pain during and after pregnancy: a systematic review and meta-analysis. J Bodyw Mov Ther. 2017;21(4):752-62.

17. Garland EL. Treating chronic pain: the need for non-opioid options. Expert Rev Clin Pharmacol. 2014;7(5):545-50.

18. Lao L. Acupuncture techniques and devices. J Altern Complement Med. 1996;2(1):23-5.

19. Wilkinson J, Faleiro R. Acupuncture in pain management. Contin Educ Anaesth Crit Care Pain. 2007;7(4):135-8.

20. Lim T-K, Ma Y, Berger F, Litscher G. Acupuncture and neural mechanism in the management of low back pain-an update. Medicines. 2018;5(3):63.

21. Thomas KJ, MacPherson $\mathrm{H}$, Thorpe L, Brazier J, Fitter M, Campbell MJ, Roman M, Walters SJ, Nicholl J. Randomised controlled trial of a short course of traditional acupuncture compared with usual care for persistent non-specific low back pain. BMJ. 2006;333(7569):623.

22. Cho Y-J, Song Y-K, Cha Y-Y, Shin B-C, Shin I-H, Park H-J, Lee H-S, Kim K-W, Cho J-H, Chung W-S, Lee J-H, Song M-Y. Acupuncture for chronic low back pain: a multicenter, randomized, patientassessor blind, sham-controlled clinical trial. Spine. 2013;38(7):549-57.

23. Pach D, Yang-Strobel X, Lüdtke R, Roll S, Icke K, Brinkhaus B, Witt CM. Standardized versus individualized acupuncture for chronic low back pain: a randomized controlled trial. Evid Based Complement Altern Med. 2013;2013:125937.

24. Zhang X, Wang Y, Wang Z, Wang C, Ding W, Liu Z. A randomized clinical trial comparing the effectiveness of electroacupuncture versus medium-frequency electrotherapy for discogenic sciatica. Evid Based Complement Altern Med. 2017;2017: 9502718.

25. Shin J-Y, Ku B, Kim JU, Lee YJ, Kang JH, Heo H, Choi H-J, Lee J-H. Short-term effect of laser acupuncture on lower back pain: a randomized, placebo-controlled, double-blind trial. Evid Based Complement Altern Med. 2015;2015:808425.

26. Foster NE, Bishop A, Bartlam B, Ogollah R, Barlas P, Holden M, Ismail K, Jowett S, Kettle C, Kigozi J, 
Lewis M, Lloyd A, Waterfield J, Young J. Evaluating acupuncture and standard care for pregnant women with back pain (EASE Back): a feasibility study and pilot randomised trial. Health Technol Assess. 2016;20(33):1-236.

27. Bhardwaj A, Nagandla K. Musculoskeletal symptoms and orthopaedic complications in pregnancy: pathophysiology, diagnostic approaches and modern management. Postgrad Med J. 2014;90(1066): 450-60.

28. Liddle SD, Pennick V. Interventions for preventing and treating low-back and pelvic pain during pregnancy. Cochrane Database Syst Rev. 2015;2015(9):CD001139. https://doi.org/10.1002/ 14651858.CD001139.pub4.

29. Smith CA, Collins CT, Crowther CA, Levett KM. Acupuncture or acupressure for pain management in labour. Cochrane Database Syst Rev. 2011;(7): CD009232. https://doi.org/10.1002/14651858. CD009232.

30. Schlaeger JM, Gabzdyl EM, Bussell JL, Takakura N, Yajima H, Takayama M, Wilkie DJ. Acupuncture and Acupressure in Labor. J Midwifery Womens Health. 2017;62(1):12-28.

31. Linde K, Allais G, Brinkhaus B, Fei Y, Mehring M, Vertosick EA, Vickers A, White AR. Acupuncture for the prevention of episodic migraine. Cochrane Database Syst Rev. 2016;6:CD001218.

32. Allais G, Sinigaglia S, Airola G, Rolando S, De Lorenzo C, Chiarle G, Benedetto C. Ear acupuncture in the prophylactic treatment of chronic migraine. Neurol Sci. 2019;40(Suppl 1):211-2. https://doi.org/ 10.1007/s10072-019-03800-y.

33. Nie L, Cheng J, Wen Y, Li J. The effectiveness of acupuncture combined with tuina therapy in patients with migraine. Complement Med Res. 2019;26(3):182-94. https://doi.org/10.1159/ 000496032 .

34. Pei P, Liu L, Zhao L-P, Qu Z-Y, Tang C-Y, Wang L-P, Yang W. Electroacupuncture exerts an anti-migraine effect via modulation of the 5-HT7 receptor in the conscious rat. Acupunct Med. 2019;37(1): 47-54. https://doi.org/10.1136/acupmed-2017011410.

35. Gu T, Lin L, Jiang Y, Chen J, D'Arcy RC, Chen M, Song $\mathrm{X}$. Acupuncture therapy in treating migraine: results of a magnetic resonance spectroscopy imaging study. J Pain Res. 2018;11:889-900.

36. Kim J-M, Jeon H-J, Kim H-J, Cho C-K, Yoo H-S. Bee venom pharmacopuncture: an effective treatment for complex regional pain syndrome. J pharmacopuncture. 2014;17(4):66-9.
37. Mazzardo-Martins L, Salm DC, Winkelmann-Duarte EC, Ferreira JK, Lüdtke DD, Frech KP, Belmonte LAO, Horewicz VV, Piovezan AP, Cidral-Filho FJ, Moré AOO, Martins DF. Electroacupuncture induces antihyperalgesic effect through endothelin-B receptor in the chronic phase of a mouse model of complex regional pain syndrome type I. Pflüg Arch Eur J Physiol. 2018;470(12):1815-27.

38. Wang C, Li K, Choudhury A, Gaylord S. Trends in yoga, tai chi, and qigong use among US adults, 2002-2017. Am J Public Health. 2019;109(5): 755-61.

39. Weifen W, Muheremu A, Chaohui C, Wenge L, Lei $S$. Effectiveness of tai chi practice for non-specific chronic low back pain on retired athletes: a randomized controlled study. J Musculoskelet Pain. 2013;21(1):37-45.

40. Hall AM, Maher CG, Lam P, Ferreira M, Latimer J. Tai chi exercise for treatment of pain and disability in people with persistent low back pain: a randomized controlled trial. Arthritis Care Res. 2011;63(11):1576-83.

41. Zou L, Zhang Y, Liu Y, Tian X, Xiao T, Liu X, Yeung AS, Liu J, Wang X, Yang Q. The effects of tai chi chuan versus core stability training on lower-limb neuromuscular function in aging individuals with non-specific chronic lower back pain. Medicina. 2019;55(3):60.

42. Liu J, Yeung A, Xiao T, Tian X, Kong Z, Zou L, Wang $X$. Chen-style tai chi for individuals (aged 50 years old or above) with chronic non-specific low back pain: a randomized controlled trial. Int J Environ Res Public Health. 2019;16(3):517.

43. Moore AA, Lake JE, Glasner S, Karlamangla A, Kuerbis A, Preciado D, Jenkins J, Dominguez BX, Candelario J, Liao DH, Tang L, Reid MC. Establishing the feasibility, acceptability and preliminary efficacy of a multi-component behavioral intervention to reduce pain and substance use and improve physical performance in older persons living with HIV. J Subst Abuse Treat. 2019;100: 29-38.

44. Burschka JM, Keune PM, Oy UH, Oschmann P, Kuhn P. Mindfulness-based interventions in multiple sclerosis: beneficial effects of tai chi on balance, coordination, fatigue and depression. BMC Neurol. 2014;14:165.

45. Taylor E, Taylor-Piliae RE. The effects of tai chi on physical and psychosocial function among persons with multiple sclerosis: a systematic review. Complement Ther Med. 2017;31:100-8.

46. Tavee J, Rensel M, Planchon SM, Butler RS, Stone L. Effects of meditation on pain and quality of life in 
multiple sclerosis and peripheral neuropathy: a pilot study. Int J MS Care. 2011;13(4):163-8.

47. Tsai P-F, Kitch S, Chang JY, James GA, Dubbert P, Roca JV, Powers CH. Tai chi for posttraumatic stress disorder and chronic musculoskeletal pain: a pilot study. J Holist Nurs. 2018;36(2):147-58.

48. Grodin MA, Piwowarczyk L, Fulker D, Bazazi AR, Saper RB. Treating survivors of torture and refugee trauma: a preliminary case series using qigong and t'ai chi. J Altern Complement Med. 2008;14(7): 801-6.

49. Slattengren AH, Nissly T, Blustin J, Bader A, Westfall E. Best uses of osteopathic manipulation. J Fam Pract. 2017;66(12):743-7.

50. Clinical Guideline Subcommittee on Low Back Pain, American Osteopathic Association. American Osteopathic Association guidelines for osteopathic manipulative treatment (OMT) for patients with low back pain. J Am Osteopath Assoc. 2010;110(11): 653-66.

51. Licciardone JC, Gatchel RJ, Aryal S. Recovery from chronic low back pain after osteopathic manipulative treatment: a randomized controlled trial. J Am Osteopath Assoc. 2016;116(3):144-55.

52. Cerritelli F, Ginevri L, Messi G, Caprari E, Di Vincenzo M, Renzetti C, Cozzolino V, Barlafante G,
Foschi N, Provinciali L. Clinical effectiveness of osteopathic treatment in chronic migraine: 3 -armed randomized controlled trial. Complement Ther Med. 2015;23(2):149-56.

53. Goertz CM, Salsbury SA, Long CR, Vining RD, Andresen AA, Hondras MA, Lyons KJ, Killinger LZ, Wolinsky FD, Wallace RB. Patient-centered professional practice models for managing low back pain in older adults: a pilot randomized controlled trial. BMC Geriatr. 2017;17(1):235.

54. Moore C, Adams J, Leaver A, Lauche R, Sibbritt D. The treatment of migraine patients within chiropractic: analysis of a nationally representative survey of 1869 chiropractors. BMC Complement Altern Med. 2017;17(1):1-10.

55. Chaibi A, Benth J, Tuchin PJ, Russell MB. Chiropractic spinal manipulative therapy for migraine: a three-armed, single-blinded, placebo, randomized controlled trial. Eur J Neurol. 2017;24(1):143-53.

56. Bussières AE, Stewart G, Al-Zoubi F, Decina $P$, Descarreaux M, Hayden J, Hendrickson B, Hincapié C, Pagé I, Passmore S, Srbely J, Stupar M, Weisberg J, Ornelas J. The treatment of neck pain-associated disorders and whiplash-associated disorders: a clinical practice guideline. J Manip Physiol Ther. 2016;39(8):523-564.e27. 